\title{
Localized States for Elementary Systems
}

\author{
T. D. Newton and E. P. Wigner \\ Palmer Physical Laboratory, Princeton University, Princeton, New Jersey
}

\begin{abstract}
It is attempted to formulate the properties of localized states on the basis of natural invariance requirements. Chief of these is that a state, localized at a certain point, becomes, after a translation, orthogonal to all the undisplaced states localized at that point. It is found that the required properties uniquely define the set of localized states for elementary systems of non-zero mass and arbitrary spin. The localized functions belong to a continuous spectrum of an operator which it is natural to call the position operator. This operator has automatically the property of preserving the positive energy character of the wave function to which it is applied (and it should be applied only to such wave functions). It is believed that the development here presented may have applications in the theory of elementary particles and of the collision matrix.
\end{abstract}

\section{INTRODUCTION}

$I^{T}$ $\mathrm{T}$ is well known that invariance arguments suffice to obtain an enumeration of the relativistic equations for elementary systems. ${ }^{1}$ The concept of an "elementary system" is, however, not quite identical with the intuitive concept of an elementary particle. Intuitively, we consider a particle "elementary" if it does not appear to be useful to attribute a structure to it. The definition under which the aforementioned enumeration can be made is a more explicit one: it requires that all states of the system be obtainable from the relativistic transforms of any state by superpositions. In other words, there must be no relativistically invariant distinction between the various states of the system which would allow for the principle of superposition. This condition is often referred to as irreducibility condition. Relativistic transform is meant to include in the above connection not only the customary Lorentz transformations but also rotations and displacements in space and time.

The role of elementary systems as initial and final states of collision phenomena, and hence their connection with the theory of the collision matrix, will be discussed at the end of this article. We wish to turn now to the connection of elementary systems with elementary particles.

Two conditions seem to play the most important role in the concept of an elementary particle. The first one is that its states shall form an elementary system in the sense given above. This condition is quite unambiguous. The second condition is less clear cut: it is that it should not be useful to consider the particle as a union of other particles. In the case of an electron or a proton both conditions are fulfilled and there is no question as to the elementary nature of these particles. Only the first condition is fulfilled for a hydrogen atom in its normal state and we do not consider it to be an elementary particle.

The situation is more ambiguous, for instance, in the

1 E. P. Wigner, Ann. of Math. 40, 149 (1939). The concept of an elementary system, which will be explained below, is a description of a set of states which forms, in mathematical language, an irreducible representation space for the inhomogeneous Lorentz group. case of a $\pi$-meson. Qualitatively, a $\pi$-meson differs in no way from a very sharp resonance state, formed by the collision of a $\mu$-meson and a neutrino. Strictly speaking, the states of a $\pi$-meson do not form an elementary system because, after a sufficiently long time, it can be either in the dissociated or in the undissociated state and the distinction between these is surely invariant relativistically. Nevertheless, the life time of the $\pi$-meson is very long as compared with any relevant unit of time (such as $h / m c^{2}$ ) and within this time interval its states do form an elementary system. On the other hand, the properties of a $\pi$-meson are very different from what one would expect from a compound consisting of a $\mu$-meson and a neutrino. Thus the second condition for an elementary particle is fulfilled. It is this condition which has no counterpart in the definition of an elementary system. As a result of this circumstance the concept of an elementary system is much broader than that of an elementary particle; as was mentioned above, a hydrogen atom in its normal state forms an elementary system.

Every system, even one consisting of an arbitrary number of particles, can be decomposed into elementary systems. These elementary systems can be specified in a relativistically invariant manner, as containing only certain states. Thus, the restriction to the normal state of the hydrogen atom selected an elementary system from all the states of the hydrogen atom, which, together, do not form an elementary system. The usefulness of the decomposition into elementary systems depends on how often one has to deal with linear combinations containing several elementary systems.

The great drawback of using the elementary systems as a basis of the theory is that their existence follows by a rather abstract argument from the principles of quantum mechanics. As a result, the expressions for some of the most important operators "get lost" in the process. The only physical quantities for which the theory directly provides expressions are the basic quantities of the components of the momentum-energy vector and the six components of the relativistic angular momentum tensor. The subject of the present article is an attempt to find general, invariant theoretic principles 
on the basis of which operators for the position coordinates can be found.

If we restrict ourselves to an elementary system, the physical interpretation of the operators to be found is unique: they will correspond to the position of the particle if we deal with an elementary particle. Otherwise they may correspond to the center of mass of the system. If the system is not elementary, the interpretation will not be unique and neither will our postulates lead us to a uniquely determined set of operators.

Before proceeding with our argument, we wish to refer to other investigations with somewhat similar objectives. The problem of the center of mass in relativity theory has been treated particularly by Eddington ${ }^{2}$ and by Fokker ${ }^{3}$ on the basis of non-quantum mechanics. Their work was evaluated and a quantum mechanical generalization thereto given by Pryce. ${ }^{4} \mathrm{We}$ shall have frequent occasion to refer to his results. Ideas related to Pryce's work have been first put forward by Schrödinger ${ }^{5}$ and, more recently, by Finkelstein $^{6}$ and also by M $\phi$ ller. $^{7}$

The present paper arose from a reinvestigation of the irreducible representations ${ }^{8}$ of de Sitter space which was undertaken by one of us. ${ }^{9}$ These representations are in a one to one correspondence with relativistically invariant wave equations for elementary systems in de Sitter space. At the conclusion of the investigation it appeared that the physical content of the equations which were obtained could be understood much more readily if position operators could be defined on an invariant theoretic basis. As an introduction to this, a similar investigation was undertaken in flat space with the results given in the following sections.

\section{POSTULATES FOR LOCALIZED STATES AND POSITION OPERATORS}

The position operator could easily be written down if the wave function of the state (or the states) were known for which the three space coordinates are zero at $t=0$. If $\psi$ is such a function and $T(a)$ the operator of displacement by $a_{x}, a_{y}, a_{z}, a_{t}$, the wave function $T(a)^{-1} \psi$ represents a state for which the space coordinates are $a_{x}, a_{y}, a_{z}$ at time $a_{t}$ : Thus the knowledge of the wave functions corresponding to the state $x=y=z$ $=0$ at $t=0$ (and the knowledge of the displacement operators) entails the knowledge of all localized states, i.e., of all characteristic functions of the position operators. From these, the position operators are easily obtained. For this reason we concentrated on obtaining

${ }^{2}$ A. S. Eddington, Fundamental Theory (Cambridge University Press, London, 1946).

3 A. D. Fokker, Relativitatstheorie (Groningen, Noordhoff, 1929).

${ }^{4}$ M. H. L. Pryce, Proc. Roy. Soc. 195A, 62 (1948).

5 E. Schrödinger, Berl. Ber. 418 (1930); 63 (1931).

${ }^{6}$ R. J. Finkelstein, Phys. Rev. 74, 1563A (1948).

${ }^{7}$ Chr. M $\phi l l e r$, Comm. Dublin Inst. for Adv. Studies A, No. 5 (1949); also A. Papapetrou, Acad. Athens 14, 540 (1939).

${ }^{8} \mathrm{~L}$. H. Thomas, Ann. of Math. 42, 113 (1941).

9 T. D. Newton, Princeton Dissertation (1949). the wave functions of those states which are, at time $t=0$, localized at the origin of the coordinate system.

We postulate that the states which represent a system localized at time $t=0$ at $x=y=z=0$ : (a) form a linear set $S_{0}$, i.e., that the superposition of two such localized states be again localized in the same manner; (b) that the set $S_{0}$ be invariant under rotations about the origin and reflections both of the spatial and of the time coordinate; (c) that if a state $\psi$ is localized as above, a spatial displacement of $\psi$ shall make it orthogonal to all states of $S_{0}$; (d) certain regularity conditions, amounting essentially to the requirement that all the infinitesimal operators of the Lorentz group be applicable to the localized states, will be introduced later.

It is to be expected that the states localized at a certain point have the same properties as characteristic functions of a continuous spectrum, i.e., they will not be square integrable but the limits of square integrable functions. It seems to us that the above postulates are a reasonable expression for the localization of the system to the extent that one would naturally call a system unlocalizable if it should prove to be impossible to satisfy these requirements.

We shall carry out our calculations in the realization of the elementary systems which was described by Bargmann and Wigner $^{10}$ and will proceed with the calculation.

\section{Particle with no spin (Klein-Gordon particle)}

The determination of the localized state is particularly simple in this case. It will be carried out in some detail in spite of this, because the same steps occur in the consideration of systems with spin.

The wave functions are defined, in this case, on the positive shell of a hyperboloid $p_{0}{ }^{2}=p_{1}{ }^{2}+p_{2}{ }^{2}+p_{3}{ }^{2}+\mu^{2}$ and we shall use $p^{1}, p^{2}, p^{3}$ as independent variables. In any formula, $p_{0}$ is an abbreviation for $\left(p_{1}^{2}+p_{2}^{2}+p_{3}^{2}\right.$ $\left.+\mu^{2}\right)^{\frac{1}{2}}$. The invariant scalar product is

$$
(\psi, \phi)=\iiint \psi\left(p_{1} p_{2} p_{3}\right)^{*} \phi\left(p_{1} p_{2} p_{3}\right) d p_{1} d p_{2} d p_{3} / p_{0}
$$

The wave function $\Phi$ in coordinate space becomes

$$
\begin{aligned}
\Phi\left(x^{1}, x^{2}, x^{3}, x^{0}\right)=(2 \pi)^{-\frac{3}{2}} & \int \phi\left(p_{1}, p_{2}, p_{3}\right) \\
& \times \exp (-i\{x, p\}) d p_{1} d p_{2} d p_{3} / p_{0}
\end{aligned}
$$$$
\text { where }
$$

$$
\begin{aligned}
\{x, p\}=x^{0} p^{0}-x^{1} p^{1}-x^{2} p^{2}-x^{3} p^{3} & \\
& =x_{0} p_{0}-x_{1} p_{1}-x_{2} p_{2}-x_{3} p_{3},
\end{aligned}
$$

is the Lorentz invariant scalar product. Throughout this paper, the covariant and contravariant components are equal for the time (0) coordinate, oppositely equal for the space $(1,2,3)$ coordinates. This governs the

${ }^{10}$ V. Bargmann and E. P. Wigner, Proc. Nat. Acad. Sci. 34, 211 (1948). 
raising and lowering of all indices. Occasionally, we shall use for the scalar product of two space-like vectors the notation $(x, p)$ so that, e.g., $\{x, p\}=x^{0} p^{0}-(x \cdot p)$.

The linear manifolds which are invariant with respect to rotations about $p_{1}=p_{2}=p_{3}=0$ are, for any integer $j$, the $2 j+1$ functions

$$
P_{m}^{j}(\vartheta, \phi) f(p) \quad(m=-j,-j+1, \cdots j-1, j),
$$

where $p, \vartheta$ and $\phi$ are polar coordinates for $p_{1}, p_{2}, p_{3}$ and $f$ is an arbitrary function. The $P_{m}^{i}$ are the well-known spherical harmonics. The sets (4) are also invariant with respect to inversion, i.e., replacement of $p_{1}, p_{2}, p_{3}$ by $-p_{1},-p_{2},-p_{3}$. Naturally, not only a single set (4) has these properties of invariance but the sum of an arbitrary number of such sets as long as one includes with one function (4) all $2 j+1$ functions and their linear combinations. The $f(p)$ could be different for different $j$.

Under time reversal $\psi\left(p_{1} p_{2} p_{3}\right)$ goes over into ${ }^{11}$

$$
\Theta \psi\left(p_{1}, p_{2}, p_{3}\right)=\psi\left(-p_{1},-p_{2},-p_{3}\right)^{*} \text {. }
$$

We understand by time reversal the operation which makes out of a wave function $\psi$ the wave function $\theta \psi$ on which every experiment, if carried out at $-t$, yields same results as the same experiment carried out on $\psi$ at time $t$. Because of (5) and our postulate (b), if the $P_{m}^{j}(\theta, \phi) f(p)$ are localized at the origin, the set $P_{-m}{ }^{j}(\theta, \phi) f(p)^{*}$, i.e., the $P_{+m}{ }^{j}(\theta, \phi) f(p)^{*}$ are also localized. The same is then true for the sum and difference of the corresponding pairs of functions which shows that the $f(p)$ can be assumed to be real without loss of generality.

The displacement operator in momentum space is simply multiplication with $\exp (-i\{a, p\})$;

$$
T(a) \psi=\exp (-i\{a, p\}) \psi \text {. }
$$

We shall have to consider purely space-like displacements, i.e., assume that $a^{0}=0$. It then follows from our postulate $(c)$ that, in particular, $\exp (i(a, p)) \psi$ is orthogonal to $\psi$ if $\psi$ is localized, or that

$$
\begin{aligned}
\iiint\left|\psi\left(p_{1} p_{2} p_{3}\right)\right|^{2} \exp i\left(a_{1} p_{1}+a_{2} p_{2}\right. \\
\left.+a_{3} p_{3}\right) d p_{1} d p_{2} d p_{3} / p_{0}=0,
\end{aligned}
$$

for any non-vanishing vector $a$. This shows that only the zero wave number part occurs in the expansion of $|\psi|^{2} / p_{0}$ into a Fourier integral. Hence $|\psi|^{2} / p_{0}$ is a constant, $|\psi|$ proportional to $p_{0} \frac{1}{2}$. Comparing this with (4), we see that only the set $j=0$ can be chosen. Since, furthermore, we saw that $f(p)$ can be assumed to be real, we have

$$
\psi^{2}=(2 \pi)^{-3} p_{0}
$$

As was anticipated, $(\psi, \psi)$ is infinite, the localized function is part of a continuous spectrum.

\footnotetext{
${ }^{11}$ E. P. Wigner, Gottinger Nachrichten 546 (1932).
}

As far as postulates $(a),(b),(c)$ are concerned, $\psi$ could be a discontinuous function, being $+p_{0^{\frac{1}{2}}}=\left(p^{2}+\mu^{2}\right)^{\frac{1}{4}}$ for some $p$, and $-\left(p^{2}+\mu^{2}\right)^{\frac{1}{2}}$ for the remaining $p$. However, no matter how $\psi$ is chosen, consistently with (7), there is, in this case, only one state localized at the origin because if there were two, say $\psi_{1}$, and $\psi_{2}$, the $\psi_{1}$ would have to be orthogonal not only to $\psi_{1} \exp (-i(a, p))$ but also to $\psi_{2} \exp (-i(a, p))$ from which not only $|\psi|^{2} \sim p_{0}$ but also $\psi_{1}^{*} \psi_{2} \sim p_{0}$ and hence the proportionality of $\psi_{1}$ to $\psi_{2}$ follows.

In order to eliminate the discontinuous $\psi$ as localized state, we introduce the further regularity condition that

$$
\left(M^{0 k} \psi_{n}, M^{0 k} \psi_{n}\right) /\left(\psi_{n}, \psi_{n}\right)
$$

shall remain finite as the normalizable wave functions $\psi_{n}$ approach $\psi$. The $M_{0 k}$ is the infinitesimal operator of a proper Lorentz transformation in the $x^{0} x^{k}$ plane, its operator is ${ }^{10}$

$$
M^{0 k}=i p^{0} \partial / \partial p_{k} .
$$

This further postulate eliminates all discontinuous $\psi$ and we obtain for the wave function of the only state which is localized at the origin

$$
\psi=(2 \pi)^{-\frac{3}{2}} p_{0^{\frac{1}{2}}} .
$$

The regularity requirement $(d)$ actually asks for the finiteness of (8) for all $M^{k l}$. However, if one substitutes $M^{23}, M^{31}$ or $M^{12}$ for $M^{0 k}$ in (8), the resulting expression is automatically bounded-in fact their sum is $j(j+1)$. Hence requiring the applicability of the $M^{23}, M^{31}, M^{12}$ to $\psi$ does not introduce a new condition.

The localized wave function in coordinate space is obtained by (2). It is, apart from a constant ${ }^{12}$

$$
\Psi(r)=(\mu / r)^{5 / 4} H_{5 / 4}{ }^{(1)}(i \mu r) .
$$

It goes to zero at $r=\infty$ as $e^{-\mu r}$, at $r=0$ it becomes infinite as $r^{-5 / 2}$. It is, of course, not square integrable since it is part of a continuous spectrum.

Applying the operator of displacement to (9) we obtain for the wave function of the state which is localized at $x^{1}, x^{2}, x^{3}$ at time $t=0$

$$
\begin{aligned}
T(-x) \psi=(2 \pi)^{\frac{3}{2}} p_{0}^{\frac{1}{2}} \exp -i\left(p^{1} x^{1}+p^{2} x^{2}+p^{3} x^{3}\right) & =(2 \pi)^{-\frac{3}{2}} p_{0}^{\frac{1}{2}} e^{-i(p \cdot x)} .
\end{aligned}
$$

This must be a characteristic function of the operator $q^{k}$ for the $k$-coordinate with the characteristic value $x^{k}$. The operator $q^{k}$ is therefore defined by

$$
\begin{aligned}
q^{k} \phi(p)=(2 \pi)^{-3} \int p_{0} \frac{1}{2} e^{-i(p \cdot x)} & x^{k}\left(p_{0}\right)^{\frac{1}{2}} \\
& \times e^{i\left(p^{\prime} \cdot x\right)} \phi\left(p^{\prime}\right) d x d p^{\prime} / p_{0}{ }^{\prime} ;
\end{aligned}
$$

$d x$ and $d p^{\prime}$ stand for $d x^{1} d x^{2} d x^{3}$ and $d p_{1}{ }^{\prime} d p_{2}{ }^{\prime} d p_{3}{ }^{\prime}$. One

${ }^{12}$ G. A. Campbell and R. M. Foster, "Fourier Integrals for Practical Applications," American Telephone and Telegraph Company (1931). 
can transform (10) in a well-known fashion

$$
\begin{aligned}
q^{k} \phi(p)= & \left(i \frac{\partial}{\partial p^{k}}-\frac{i}{2} \frac{p^{k}}{p_{0}^{2}}\right)(2 \pi)^{-3} \\
& \quad \times \int p_{0}^{\frac{1}{2}} e^{i\left(p^{\prime}-p\right) \cdot x}\left(p_{0}^{\prime}\right)^{-\frac{1}{2}} \phi\left(p^{\prime}\right) d x d p^{\prime} \\
= & -i\left(\frac{\partial}{\partial p_{k}}+\frac{p^{k}}{2 p_{0}^{2}}\right) \phi(p) .
\end{aligned}
$$

These expressions are valid for finite as well as for vanishing rest mass. It is remarkable that the operator $q^{k}$ can be transformed into coordinate space and retains a relatively simple form

$$
q^{k} \Phi(x)=x^{k} \Phi(x)+\frac{1}{8 \pi} \int \frac{\exp (-\mu|x-y|)}{|x-y|} \frac{\partial \Phi(y)}{\partial y_{k}} d y .
$$

$x$ and $y$ stand for the spatial part of the four vectors $x^{\mu}$ and $y^{\mu}$ and $d y$ indicates integration over $y^{1}, y^{2}, y^{3}$. The customary $q^{k}$ operator contains only the first term of (11).

It may be well to remember at this point that the position operators to which our postulates lead necessarily commute with each other so that only Pryce's case $(e)$ can be used for comparison. In fact, our $q^{k}$ is identical with his $\tilde{q}^{k}$. It may be pointed out, second, that a state which is localized at the origin in one coordinate system, is not localized in a moving coordinate system, even if the origins coincide at $t=0$. Hence our operators $q^{k}$ have no simple covariant meaning under relativistic transformations. This is not the case for the customary operators $q^{k}$ either. Furthermore, even though it appears that $\Phi(x)=\delta(x)$ is invariant under relativistic transformations which leave the origin unchanged, this is not much more than a mathematical quirk. One sees this best by transforming the $\delta$-function to momentum space through the inversion of (2). The result, $p_{0}$, seems to have a simple covariant meaning. However, it does not represent a square integrable function and if one approximates it by one, say by $\psi_{\alpha}=p_{0} \exp \left(-\alpha^{2} p_{0}^{2}\right)$, the Lorentz transform of $\psi_{\alpha}$ will not approach $\psi_{\alpha}$ with decreasing $\alpha$. In fact, as soon as $\alpha \mu \ll 1$, the scalar product of $\psi_{\alpha}$ and its transform will be independent of $\alpha$ and smaller than the norm of $\psi_{\alpha}$.

\section{Particles with spin and finite mass}

We again use the description given in reference 10 , i.e., define wave functions on the positive hyperboloid $p_{0}{ }^{2}=p_{1}{ }^{2}+p_{2}{ }^{2}+p_{3}{ }^{2}+\mu^{2}$ and use in addition to $p_{1}, p_{2}, p_{3}$ the $2 s$ spin variables $\xi_{1}, \xi_{2}, \cdots \xi_{2 s}$ all of them fourvalued. The wave functions which describe the possible states of the system will be symmetric functions of the $\xi$ and satisfy the $2 s$ equations

$$
\sum_{\kappa} \gamma_{\alpha}{ }^{k} p_{k} \psi=\mu \psi \quad \alpha=1,2, \cdots 2 s .
$$

The consistency of these was shown before. ${ }^{10}$ The $\gamma_{\alpha}$ apply to $\xi_{\alpha}$, two $\gamma$ with different first indices commute, with the same first index they satisfy the well-known relations

$$
\gamma_{\alpha}{ }^{\kappa} \gamma_{\alpha}{ }^{\lambda}+\gamma_{\alpha}{ }^{\lambda} \gamma_{\alpha}{ }^{\kappa}=2 g^{\kappa \lambda}
$$

The great difference between the present case and that of zero spin consists in the limitation (13) of the permissible wave functions, in addition to the limitation to the positive hyperboloid. This latter limitation can be taken care of by using only the $p_{1}, p_{2}, p_{3}$ as independent variables, the former limitation cannot be taken care of in an equally simple fashion. We shall make extensive use, however, of a device, most successfully employed by Schrödinger ${ }^{5}$ and define operators

$$
E_{\alpha}=\frac{1}{2}\left(p_{0}\right)^{-1}\left(\sum_{\kappa} \gamma_{\alpha}{ }^{k} p_{\kappa}+\mu\right) \gamma_{\alpha}{ }^{0} .
$$

This is a projection operator: $E_{\alpha}{ }^{2}=E_{\alpha}$ and $E_{\alpha} \psi$ automatically satisfies the corresponding Eq. (13). Denoting the product of all the $E_{\alpha}$ by $E$

$$
E=E_{1} E_{2} \cdots E_{2 s}
$$

any $E \psi$ is a permissible wave function, satisfying all Eqs. (13).

For the scalar product, we shall use the expression

$$
(\psi, \phi)=\int p_{0}^{-2 s-1} \sum_{\xi} \psi^{*} \phi d p
$$

It follows from this at once, because of our postulate (c), and since (6) is valid in this case also, that every wave function which is localized at the origin satisfies the analogon of $(7)$ :

$$
\sum_{\xi}|\psi|^{2}=(2 \pi)^{-3} p_{0}^{2 s+1}
$$

The operator for time reversal is

$$
\Theta \psi\left(p_{1}, p_{2}, p_{3}\right)=C \psi\left(-p_{1},-p_{2},-p_{3}\right)^{*},
$$

where $C$ is a matrix which operates on the $\xi$ coordinates and satisfies the equations

$$
\begin{array}{ll}
C \gamma_{\alpha}{ }^{* *}=\gamma_{\alpha}{ }^{0} C ; & (\alpha=1,2, \cdots, 2 s) \\
C \gamma_{\alpha}{ }^{k *}=-\gamma_{\alpha}{ }^{k} C . & (\alpha=1,2, \cdots, 2 s ; k=1,2,3) .
\end{array}
$$

If $\gamma^{0}, \gamma^{2}, \gamma^{3}$ are real, $\gamma^{1}$ imaginary

$$
C=\prod_{\alpha=1}^{2 s} \gamma_{\alpha}^{2} \gamma_{\alpha}^{3} ; \quad C^{2}=(-)^{2 s}
$$

Since $C$, as defined above, is a real matrix we also have $\Theta^{2}=(-)^{2 s}$ which is true independently of the choice of the $\gamma$-matrices. The operator for the inversion of the space coordinates is

$$
I \psi\left(p_{1}, p_{2}, p_{3}\right)=\gamma_{1}{ }^{0}{\gamma_{2}}^{0} \cdots \gamma_{2 s}{ }^{0} \psi\left(-p_{1},-p_{2},-p_{3}\right) ;
$$

it commutes with the $E_{\alpha}$ of (14).

In order to determine the sets of wave functions which are invariant under rotations, we first define the analogue of the pure spin function for the relativistic 
Eqs. (13). For this purpose we define auxiliary functions $v_{m}$ which are independent of $p_{1}, p_{2}, p_{3}$ and functions of the $\xi$ only. They satisfy the equations

and

$$
\gamma_{\alpha}^{0} v_{m}=v_{m} \quad(\alpha=1,2, \cdots, 2 s)
$$

$\frac{1}{2} i \sum_{\alpha} \gamma_{\alpha}^{1} \gamma_{\alpha}^{2} v_{m}=m v_{m}$

$$
(m=-s,-s+1, \cdots, s-1, s) .
$$

Since the $\gamma^{0}$ and the $i \gamma^{1} \gamma^{2}$ commute, it is possible to assume temporarily that they are all diagonal. Equation (19) then demands that they all belong to the characteristic value +1 of each $\gamma^{0}$; there are $2^{2 s}$ such functions. However, we are interested only in symmetric functions of the $\xi$ and there are only $2 s+1$ of these. They are distinguished by the index $m$ : the $v_{m}$ has non-zero components only for those $\xi$ for which $s+m$ of the $i \gamma^{1} \gamma^{2}$ are +1 , the remaining $s-m$ are -1 . For these $\xi$ the value of $v_{m}$ is $((s+m) !(s-m) ! /(2 s) !)^{\frac{1}{2}} 2^{-s}$ so that $v_{m}$ is normalized in the sense

$$
\sum_{\xi}\left|v_{m}\right|^{2}=\sum_{\xi} v_{m}^{2}=1
$$

Physically, $m$ corresponds to the spin angular momentum about the $x^{3}$ axis, the parity of $v_{m}$ is even because of (19) and (18).

The $v_{m}$ are not permissible wave functions because they do not satisfy the wave Eqs. (13). We therefore define as spin functions

$V_{m}\left(p_{1}, p_{2}, p_{3}, \xi_{1}, \cdots, \xi_{2 s}\right)=E v_{m} \quad(m=-s, \cdots, s)$.

They are permissible wave functions of even parity and $V_{m}$ represents a state of angular momentum $m \hbar$ about the third coordinate axis. Their normalization is, instead of $(19 \mathrm{~b})$

$$
\sum_{\xi}\left|V_{m}\right|^{2}=\left(\left(p_{0}+\mu\right) / 2 p_{0}\right)^{2 s} \text {. }
$$

The most general solution of (13) is a linear combination of the $V_{m}$ multiplied with arbitrary functions of the $p_{1}, p_{2}, p_{3}$. A set of wave functions which is invariant under rotations and reflections contains wave functions of the form

$$
\psi_{j m}=\sum_{l, m^{\prime}} S(l, s)_{j, m-m^{\prime}, m^{\prime}} P_{m-m^{\prime}}^{l}(\vartheta, \phi) f_{l}(p) V_{m^{\prime}} .
$$

The $p, \vartheta, \phi$ are again polar coordinates for $p^{1}, p^{2}, p^{3}$; the $f_{l}$ are arbitrary unknown functions of the length of $p$. However, if one function of the form (21) occurs in the set, all others with different $m$ but the same $f_{l}$ also occur. The summation over $l$ is to be extended over all even values between $|j-s|$ and $j+s$ if the parity of the $\psi_{j}$ is to be even, over all odd values of $l$ if the parity of $\psi_{j}$ is odd. The $S(l, s)$ are the customary coefficients ${ }^{13}$

\footnotetext{
${ }^{13}$ See e.g., E. P. Wigner, Gruppentheorie, etc. (V. Vieweg \& Sohn Braunschweig, 1931). The composition of the $V$ and the spherical harmonics $P^{l}$ to the $\psi_{j}$ is the same operation as the composition of the spin functions with a definite $S$ and the space coordinate functions with a definite $L$, to functions of both, with definite $J$. This composition is explained in Chapter XXII. The coefficients of the composition, i.e., our $S(l, s)$ are calculated p. $202 \mathrm{ff}$ (they are denoted by $s^{(L S)}$.
}

giving a total $j$ from wave functions with given "orbital momentum" $l$ and "spin momentum" $s$.

Since the polar angles $\vartheta, \phi$ are indeterminate for $p=0$, the $f_{l}(p)$ must vanish for $p=0$ unless $l=0$. Otherwise, the $\psi_{j m}$ would become singular at $p=0$ and the $M^{0 k}$ could not be applied to them in the sense of the boundedness of (8). (Actually it is necessary to postulate this equation for the square of $M^{0 k}$ instead only for $M^{0 k}$.) It follows that $\psi_{j m}$ vanishes at $p=0$ unless the series (21) contains a term with $l=0$. However, (16) shows that $\psi$ cannot vanish at this point if the rest mass is finite and that, hence, every localizable wave function must have an $l=0$ term in its expansion. This happens only if $j=s$ and the wave function has even parity. If the parity of $\psi_{j m}$ is odd, only $f_{1}, f_{3}$, etc., enter (21) and these still vanish at $p=0$. It follows that the wave functions which are localized at the origin all have angular momentum $j=s$ and the form

$$
\psi_{m}=\sum_{l=0}^{2 s} \sum_{m^{\prime}} S(l, s)_{s, m-m^{\prime}, m^{\prime}} P_{m-m^{\prime}}^{l}(\vartheta, \phi) f_{l} V_{m^{\prime}} .
$$

We now skip the part of the calculation which deals with the determination of the $f_{l}$ and give only the result: The wave functions localized at the origin are the $2 s+1$ functions

$$
\begin{aligned}
& \psi_{m}=(2 \pi)^{-3 / 2} 2^{s} p_{0}{ }^{2 s+\frac{1}{2}}\left(p_{0}+\mu\right)^{-s} \\
& \times V_{m}\left(p_{1} p_{2} p_{3} ; \xi_{1}, \cdots \xi_{2 s}\right)
\end{aligned}
$$

(i.e., the $l=0$ term alone remains from (21a)). Actually, this result is far from being surprising. ${ }^{14}$

The operator for the position coordinate can be calculated in exactly the same way as this was done in the case of zero spin, and gives

$$
q^{k}=E \prod_{\alpha=1}^{2 s}\left(1+\gamma_{\alpha}^{0}\right) \frac{p_{0}^{2 s+\frac{1}{2}}}{\left(p_{0}+\mu\right)^{s}}\left(-i \frac{\partial}{\partial p_{k}}\right) \frac{p_{0}^{-\frac{1}{2}}}{\left(p_{0}+\mu\right)^{s}} E .
$$

For $s=\frac{1}{2}$ this again agrees with Pryce's result ${ }^{4}$ for his case (e), i.e., for his operator $\tilde{q}$.

The significance of the projection operators $E$ in (22) is only to annihilate any negative energy part of the wave function to which it is applied and to produce a purely positive energy wave function. Since $q^{k}$ is the position operator only for wave functions which are

14 The proof runs as follows. One first shows, by considering $\psi_{m} \pm \Theta \psi_{-m}$ that the $f_{l}$ of (21a) can all be assumed to be real. One then subdivides $\psi_{m}$ into two parts: the $l=0$ part of the sum (21a) will be denoted by $\psi^{0}$, the rest $\psi^{r}$. As we have seen, $\psi^{0}$ is finite at $p=0$, while $\psi^{r}$ vanishes at that point. The proof then consists in showing that there can be no region in which $\psi^{r}$ is finite but very much smaller than $\psi^{0}$. It then follows from the continuity of both $\psi^{0}$ and $\psi^{r}$ that the latter vanishes everywhere. Inserting $\psi^{0}+\psi^{r}$ for $\psi$ in (16), one can neglect in the aforementioned region the square of $\psi^{r}$ as compared with the other terms. The right side, as well as the term from the square of $\psi^{0}$, are independent of $\vartheta$ and $\varphi$. This must be true, therefore, also for the term arising from the cross product of $\psi^{0}$ and $\psi^{r}$. This term is, however, a sum of expressions $P_{m}^{l}(\vartheta, \varphi) f_{0} f_{l}$ which cannot be independent of $\vartheta, \varphi$ except if all $f_{l}$ with $l>0$ vanish ( $f_{0}$ is finite by assumption). It then follows that the $f_{l}$ vanish everywhere and (21a) reduces to a single term. This can be obtained from (16) by taking the square root on both sides. 
defined on the positive hyperboloid alone, the $E$ on the right could logically be omitted. Both $E$ can be omitted $^{5}$ if one calculates a matrix element between two purely positive energy wave functions. The factors involving $p_{0}$ are necessary in order to make $i \partial / \partial p_{j}$ hermitian: because of the factor $p_{0}^{-2 s-1}$ in the volume element (15), an operator is hermitian if it looks hermitian after multiplication with $p_{0}{ }^{s+\frac{1}{2}}$ on the right and division with the same factor on the left. The operator $\prod_{a=1}^{2 s} \frac{1}{2}\left(1+\gamma_{\alpha}\right)$ is a projection operator, i.e., it is identical with its square and could therefore be inserted into (22) once more before the second $E$, thus making (22) somewhat more symmetric. The position operator (11) for the Klein-Gordon particle is a special case of (22) and can be obtained from (22) by setting $s=0$.

If one displaces a state by $a$ and measures its $x^{k}$ coordinate afterwards, the result will be greater by $a^{k}$ than the $x^{k}$ coordinate measured on the undisplaced state. This leads to the relation

$$
T(-a) q^{k} T(a)=q^{k}+a^{k}
$$

for $a^{0}=0$. Inserting the expression (6) for $T(a)$ and going to the limit of very small $a^{k}$, one obtains

$$
\left(q^{k} p^{l}-p^{l} q^{k}\right) \phi=-i \delta_{k l} \phi
$$

where $\phi$ is any permissible wave function. Actually one obtains by direct calculation, using in particular the identity

$$
E_{\alpha}\left(1+\gamma_{\alpha}^{0}\right) E_{\alpha}=p_{0}^{-1}\left(p_{0}+\mu\right) E_{\alpha}
$$

the commutation relation

$$
q^{k} p^{l}-p^{l} q^{k}=-i \delta_{k l} E
$$

The commutation relations of the $q^{k}$ with $p_{0}$ are naturally also the usual ones as $p_{0}$ is a function of the $p^{k}$ alone. Since the $q^{k}$ are the components of a vector operator in three-dimensional space, their commutation relations with the spatial components of $M^{k l}$ are also the usual ones.

We wish to remark, finally, that a consideration, similar to the above, has been carried out also for the equations with zero mass. In the case of spin 0 and $\frac{1}{2}$, we were led back to the expressions for localized systems which were given in (9) and (21b). However, for higher but finite $s$, beginning with $s=1$ (i.e., Maxwell's equations), we found that no localized states in the above sense exist. This is an unsatisfactory, if not unexpected, feature of our work. The situation is not entirely satisfactory for infinite spin either.

\section{DISCUSSION}

One might wonder, first, what the reason is that our localized states are not the $\delta$-functions in coordinate space which are usually considered to represent localized states. The reason is, naturally, that all our wave functions represent pure positive energy states. This is not true of the $\delta$-function. Similarly, our operator
(22) transforms positive energy functions into positive energy functions.

It is often stated that a measurement of the position of a particle, such as an electron, if carried out with a greater precision than the Compton wave-length, would lead to pair production and that it is, therefore, natural that the position operators do not preserve the positive energy nature of a wave function. Since a position measurement on a particle should result in a particle at a definite position, and not in a particle and some pairs, this consideration really denies the possibility of the measurement of the position of the particle. If this is accepted it still remains strange that pair creation renders the position measurement impossible to the same degree in such widely different systems as an electron, a neutron and even a neutriono. The calculations given above prove, at any rate, that there is nothing absurd in assuming the measurability of the position, and the existence of localized states, of elementary systems of non-zero mass. Moreover, the postulates $(a),(b),(c)$ and $(d)$, which are based on considerations of invariance, define the localized states and position operators uniquely for all non-zero mass elementary systems.

No similarly unique definition of localized states is possible for composite systems. Although it remains easy to show that definite total angular momenta $j$ can be attributed to localized states, one soon runs into difficulties with the rest of the argument. In particular, the summation in (16) must be extended not only over the spin coordinates $\xi$ but also over all states with different total rest mass and different intrinsic spin. As a result, one can, e.g., find states which can coexist as localized states in the sense of our axioms even though their $j$ values are different. This is also what one would expect on ordinary reasoning since, if the system contains several particles, the states in which any one of them is localized at the origin satisfy our postulates. This holds also for the states in which another one of the particles is so localized or for states in which an arbitrary linear combination of the coordinates is zero. As a result, not only is the number of localized states greatly augmented but, further, one must expect to find many such large sets for which our postulates hold, although no two sets can be considered to be localized simultaneously. In other words, each set of localized states is not only much larger for composite systems but one also has to make a choice between many sets all of which satisfy our postulates by themselves. It does not appear that one can proceed much further in the definition of localized states for composite systems without making much more specific assumptions. Naturally, one can define as localized states those, which, in any of the elementary parts of the composite system, appear localizable. It appears reasonable to assume that this definition corresponds to the center of mass of the whole system.

One may wonder, even in the case of elementary particles, whether the determination of the localized 
states and position operators has much significance. Such doubts might arise particularly strongly if one is inclined to consider the collision matrix as the future form of the theory. One must not forget, however, that the customary exposition of this theory refers only to questions about cross sections. There is another interesting set of questions referring to the position of the scattered particles: how much further back (i.e., closer to the scattering center) are they than if they had gone straight to the scattering center and then continued in the new direction without any delay. ${ }^{15} \mathrm{In}$ order to answer such questions in the relativistic region, one will need some definition of localized states for elementary systems. From this point of view it is satisfactory that the localized states could be defined without ambiguity just for these systems.

${ }^{15}$ L. Eisenbud, Princeton Dissertation (1948).

\title{
On the Space-Time Geometry of a Moving Rigid Body
}

\author{
A. D. Fokker \\ Natuurkundig Laboratorium van Teyler's Stichting, Haarlem, Nederland
}

\begin{abstract}
A simple argument is given to explain Herglotz' theorem that a rigid body has three degrees of freedom only. A new example of rigid motion is given.
\end{abstract}

$\mathbf{I}_{\mathrm{a}}^{\mathrm{N}}$ $\mathrm{N}$ this paper I propose to consider in some detail another concept modified by relativity theory, the concept of a rigid body and its motions. In physics a rigid body is to be a collection of particles keeping rigid equal distances. In the theory of invariants a particle must not be represented by a point, but, being a lasting event, it is represented by a time track. Thus a rigid body must be taken to be a collection of time tracks continuously bearing constant distances between them.

The distance of two time tracks must be conceived as the interval along a straight line intersecting both tracks at right angles, in the sense of $(1+3)$-dimensional geometry. Thus at a certain instant of one time track the vectors showing the distances to neighboring tracks will lie in a space perpendicular to the time track, and they will continue to do so at following instants.

Now let $P Q R S T$ in Fig. 1 delimit successive infinitesimal segments of a time track. The time track can be seen as generated by successive infinitesimal "revolutions." (A "revolution" in (1+3)-dimensional timespace sometimes means an acceleration.) Take the

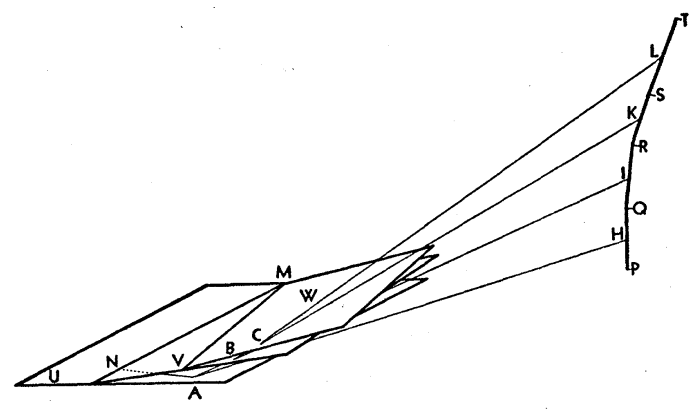

FIG. 1. spaces normal to the segments $P Q$ and $Q R$ respectively, bisecting these segments. The two spaces will intersect in a plane, $U$ say, which contains the points at equal distances from $P, Q$ and $R$. Therefore the arc $P Q R$ will be generated by an infinitesimal revolution (in $(1+3)$ dimensions) about the plane $U$. Again, the space normal to the segment $R S$ and bisecting it, will cut the bisecting space of $Q R$ in a plane $V$, and the arc $Q R S$ is generated by an infinitesimal revolution about the plane $V$. The straight intersection of $U$ and $V$ will contain the points equidistant from $P, Q, R$ and $S$. Finally the normal space bisecting the segment $S T$ will provide an intersection plane $W$ which is the "axis" of revolution generating the arc $R S T$. The intersection $M$ of the three $U, V$ and $W$ has equal distances from all five $P, Q, R, S, T$. It is the center of the osculatory equilateral superhyperboloid.

The osculatory plane containing $P Q$ and $Q R$ must be normal to the axis of revolution $U$. It is shown by $H I A$, where $A$ is the point of intersection with $U . H A$ is the principal normal of the time track, $A$ being the center of the flat hyperbole defined by $P, Q, R$. If we drop $A N$ perpendicular to $N M, N$ will be the centre of the one-blade hyperboloid defined by $P, Q, R, S$. This $A N$ is to be regarded as the binormal. Finally $N M$ is the trinormal of the time track $P Q R S T$.

When the series of infinitesimal revolutions about $U, V, W \cdots$ is generating the time track $P Q R S T \cdots$ any other points $P^{\prime}, P^{\prime \prime}$, situated in the same simultaneous space (in the same flat present, say), defined by $P$ and $U$, will generate time tracks $P^{\prime} Q^{\prime} R^{\prime} S^{\prime} T^{\prime} \cdots$, $P^{\prime \prime} Q^{\prime \prime} R^{\prime \prime} S^{\prime \prime} T^{\prime \prime} \ldots$. Two things are obvious. All these time tracks will always be normal to the flat presents $P, P^{\prime}, P^{\prime \prime} \cdots$ or $Q, Q^{\prime}, Q^{\prime \prime} \cdots$. The distances between 